\title{
MILLIMETER AND SUBMM-INTERFEROMETRY ON MT. GRAHAM, ARIZONA
}

\author{
J.H. BIEGING, R.N. MARTIN, C. WALKER, J.R.P. ANGEL, J.M. HILL, \\ N.J. WOOLF \\ Steward Observatory, University of Arizona, Tucson AZ 85721 USA
}

\author{
J.W.M. BAARS \\ Max-Planck-Institut für Radioastronomie, Auf dem Hügel 69, D-5300 \\ Bonn 1, Germany
}

\begin{abstract}
This paper draws attention to the potential for use of telescopes now under construction or planned for the near future as part of the Mt. Graham International Observatory, located in the Pinaleño Mts. east of Tucson, Arizona, for interferometer observations at mmand submm-wavelengths. The Submillimeter Telescope (SMT) should be completed by early 1993. A collaboration between the Max-PlanckInstitut für Radioastronomie and Steward Observatory, the SMT is a high-precision 10-m diameter telescope which will be useful for shortwavelength VLBI observations. A large optical telescope, consisting of two 8.4-m diameter mirrors on a common mount, is in the early design stages. When completed, it will provide for short-baseline (approx. $20-\mathrm{m}$ ) interferometry between the two optical mirrors, as well as interferometry with the SMT over baselines of a few hundred meters.
\end{abstract}

\section{THE MOUNT GRAHAM INTERNATIONAL OBSERVATORY}

Mt. Graham is the highest mountain in the Pinaleño range, some $120 \mathrm{~km}$ east of Tucson, Arizona. In November 1988, 125 acres (approx. $0.5 \mathrm{sq} . \mathrm{km}$ ) of the mountain were designated as the Mt. Graham International Observatory (MGIO) site, with provision for up to 7 telescopes to occupy up to 25 acres ( $0.1 \mathrm{sq} . \mathrm{km}$ ) of the site. At present 2 instruments-the Submillimeter Telescope and the Vatican Observatory Advanced Technology $1.8 \mathrm{~m}$ optical telescope-are nearing completion. A third-the Columbus Project-is in the detailed planning stage.

The Mt. Graham site was selected on the basis of several characteristics which are favorable for optical, infrared, and sub-mm astronomy. The site is very dark, with little light pollution from urban areas. The $3200 \mathrm{~m}$ elevation gives excellent atmospheric transparency with a high percentage of clear days and nights. There is no significant diurnal variation in cloud cover or transparency except during the summer wet season (typically 8 weeks long). There is a good access road to the site which can readily be maintained all year. Driving time from Tucson is about 3.5 hours, facilitating installation and maintenance of 
instruments.

The Arizona-MPIfR Submillimeter Telescope (SMT): Construction of the SMT is now well along and will be completed in early 1993. The telescope was built by the German firms Krupp and MAN with funding through the Max-Planck-Institut für Radioastronomie (Bonn). The enclosure building, site, and infrastructure are provided by the University of Arizona through Steward Observatory. A full complement of heterodyne receivers and bolometer arrays will be available to cover the atmospheric windows from 230 to $900 \mathrm{GHz}$.

The Columbus Telescope Project: The Columbus Project is a joint effort by the University of Arizona and the government of Italy through Arcetri Observatory, with additional funding recently made available by The Research Corporation of Tucson, Arizona. Funding is now assured and detailed design work is underway. The telescope will consist of two $8.4 \mathrm{~m}$ diameter $\mathrm{f} / 1.14$ mirrors on a common mount. The mirrors will be made in the University of Arizona Mirror Laboratory by spin-casting of borosilicate glass blanks and polishing with the stressed-lap technique. This technology-largely developed at the Mirror Laboratory-has already been used successfully to make three $1.8 \mathrm{~m}$ and three $3.5 \mathrm{~m}$ mirrors, and one $6.5 \mathrm{~m}$ mirror. A second $6.5 \mathrm{~m}$ mirror will be made in 1993, followed by the two $8.4 \mathrm{~m}$ mirrors for the Columbus Project.

\section{AND SUBMM INTERFEROMETRY POTENTIAL OF MGIO}

Very Long Baseline Interferometry: MGIO telescopes will offer possibilities for use in VLBI experiments at very short wavelengths. The SMT will have heterodyne receivers covering all atmospheric windows in the submm band. The telescope could also be used in VLBI observations at $230 \mathrm{GHz}$. With the completion of the Columbus binocular telescope, the SMT and Columbus telescopes could be used in a phased-up configuration as a single element in VLBI experiments at short-mm and submm wavelengths, with collecting area equivalent to a $15.5 \mathrm{~m}$ diameter single antenna.

Columbus Binocular 8.4m Telescope: The Columbus telescope design, with side-by-side $8.4 \mathrm{~m}$ mirrors on a common mount, is intended to provide a powerful optical and IR interferometric capability. The telescope will also be usable at submm wavelengths as an interferometer which covers an annulus in the $(u, v)$-plane between 6 and 22.8 meters projected baseline. Because the mount is alt-az, the $(u, v)$ tracks are circular (not elliptical) arcs at all declinations.

SMT-Columbus Fixed Baseline Interferometer: The SMT and Columbus telescopes will be connected by large bandwidth data links which will allow use of the telescopes as elements of an interferometer at short millimeter and sub-mm wavelengths. The precise location of the Columbus telescope is still under study, but possible sites within the MGIO reserve would give fixed baselines between the two telescopes ranging from about 150 to 700 meters, with predominantly east-west orientations. The collecting area will be equivalent to a $10-\mathrm{m}$ and 11.8-m diameter pair. 